Science and Managem

$\because \because$ International Journal of Service Science and Management

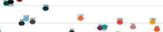

\title{
Airport Taxi Management Based on Dynamic Decision and Multi- objective Programming Model
}

\section{Hairui Zhang ${ }^{1 *}$, Zhong Zheng ${ }^{2}$, Yu Cao ${ }^{1}$}

${ }^{1}$ College of Science, China Three Gorges University, Yichang, 443002, China.

${ }^{2}$ College of Electrical Engineering \& New Energy, China Three Gorges University, Yichang, 443002, China.

\section{ABSTRACT}

Nowadays major airports often have a large number of ${ }^{*}$ Correspondence to Author: passengers and vehicles stranded, resulting in inefficient Hairui Zhang airport operations. In order to solve this problem, this article College of Science, China Three aims at the decision-making problems that airport taxi drivers Gorges University, Yichang, face after sending passengers to the airport, and the problem 443002, China.

of setting up the boarding point of the airport by analyzing the influencing mechanism of factors related to taxi driver decisions, and considering the changing rules of airport passengers and the benefits of taxi drivers, we have established Driver's choice decision model based on time periods and Multi-objective programming model based on queuing theory. The model established in this paper can greatly alleviate the airport's loadbearing pressure, improve the airport's overall boarding efficiency under the condition that the taxi driver's income is balanced, and provide a theoretical basis for the systematization of airport taxi 2020; $3: 7$ queuing.

Keywords: Multi-objective programming; Queuing theory; Periodic dynamic decision mode; Vehicle threshold

How to cite this article:

Hairui Zhang, Zhong Zheng, Yu Cao. Airport Taxi Management Based on Dynamic Decision and Multi-objective Programming Model. International Journal of Service Science and Management, eScîPUb
eSciPub LLC, Houston, TX USA.
Website: https://escipub.com/ 


\section{Introduction}

Since the reform and opening up, China's transportation industry has developed rapidly, and has become the country with the fastest development of civil aviation infrastructure construction in the world today. With the rapid development of civil aviation, more and more people choose to travel by air. With the increase in passenger flow at the airport, as one of the main means of transportation for people to and from the airport, the demand for taxis has also increased substantially. How to implement a queuing system for taxis has become a very important issue ${ }^{[1]}$.

As the airport often separates the two channels of pick-up and drop-off, taxi drivers arriving at the airport often face two choices: one is that they enter the airport's arrival area (car storage pool) to wait for passengers; the other is that they choose to return to the city empty. Drivers who choose to wait for passengers often need to pay a certain amount of time cost. They wait in the arrival area in the order of "first come, first served", and the length of waiting time often depends on both the queued taxi and the queued passengers. Taxi drivers who choose to return to the city by empty cars often need to pay costs, which include two aspects of the empty load return of empty cars and the possible loss of passenger benefits. When making a choice, taxi drivers can directly observe the number of flights in a certain period of time and the number of taxis waiting in line in the arrival area, and then make a decision based on their own experience. However, there are also many factors that will affect the decision of taxi drivers. In addition, most of the time, there are often situations where taxis line up to carry passengers and passengers line up to board. Airports often want to increase the efficiency of passengers by increasing the number of boarding points, the increase in the number will increase the possibility of traffic accidents and the maintenance and management costs of the airport. For this reason, in view of the above phenomena, this article hopes to analyze and study the influencing mechanism of factors related to taxi driver decision-making, comprehensively consider the changing rules of the number of passengers at the airport and the profit of taxi drivers, establish a taxi driver selection decision model, and give the driver selection strategy; and reasonably set up boarding points on both sides of the parallel lanes of the airport boarding area, so as to ensure the safety of vehicles and passengers, so as to maximize the overall boarding efficiency ${ }^{[2-}$ 3].

\section{Model assumptions}

Before analyzing the problem and establishing a mathematical model, we need to put forward some assumptions on the problem to simplify the problem. The assumptions made in this article are as follows:

(1) Assume that the road to or from the airport in the urban area is in good condition and there will be no long-term traffic jams and traffic accidents;

(2) Assume that passengers at the airport do not consider transportation methods such as online car booking, friend pick-up, and walking;

(3) Assume no delays in airport flights;

(4) Assume that the driver is driving at a constant speed of $50 \mathrm{~km} / \mathrm{h}$ while driving;

(5) It is assumed that the boarding time of each passenger is the same;

(6) The taxi driver returned to the city and immediately carried the passenger.

\section{Analysis of the problem}

In order to obtain a reasonable selection strate- 
gy, we first need to determine the relevant factors that affect the taxi driver's decision-making, and find the mechanism of these relevant factors on the driver's decision-making. Based on this influence mechanism to determine the relationship between the various factors, in order to establish the Driver's choice decision model.

First of all, there are only two options for taxi drivers after arriving at the airport. Plan $A$ is to go to the car storage pool to wait in line for passengers to return to the city. Plan B is to return to the city without passengers. Both schemes have advantages and disadvantages, and both need to pay a certain cost. Now we need to compare the two schemes to find out which scheme will make the most of the taxi driver's benefit in different situations. Because the passenger loading situation is different for the scenario $B$ from the airport to the city and returning from the city to the airport, the taxi is taken from the airport to the city and then returned to the airport as a carrying passengers cycle. The cycle situation reflects the benefits of the program. For plan $A$, the length of a carrying passengers cycle is longer than that of plan $B$ because $A$ spends time waiting in line for passengers, but the corresponding plan $A$ does not need to return to the city empty, so the benefit of plan $A$ in a single carrying passengers cycle is higher than plan $B$. Therefore, it is meaningless to compare the driver's benefits only once in one cycle, but to make a selection decision by comparing the benefits of the two schemes per unit of time.
For the number of boarding points at the airport, factors such as passenger efficiency, vehicle traffic safety, and management costs need to be considered. There are usually two situations at the airport. During the off-season, the passenger flow at the airport is small, and some taxi drivers may not stay at the airport waiting for passengers. At this time, there are fewer taxis and passengers in the system, and there will be no strong competition or Traffic jams do not have statistical significance; in the peak season, the airport has a large passenger flow, and taxi drivers will gather at the airport to wait for the arrival of business. At this time, there are more taxis and passengers in the system, which can be regarded as unlimited taxis. In addition, passengers are also continuously flowing into the airport boarding area. Therefore, this article takes the peak season airport as the research object. It is assumed that the taxis are unlimited and passengers are continuously flowing into the airport boarding area.

At present, the airport boarding area has two parallel lanes. How to use these two lanes and reasonably arrange boarding points is a problem for the management department to resolve. In consideration of the safety of the vehicle and passengers, it is stipulated that two parallel lanes are prohibited from crossing each other at will, and the points on both sides of the road shall be kept balanced when setting up the boarding points. The general schematic of this solution is shown in Fig. 1.

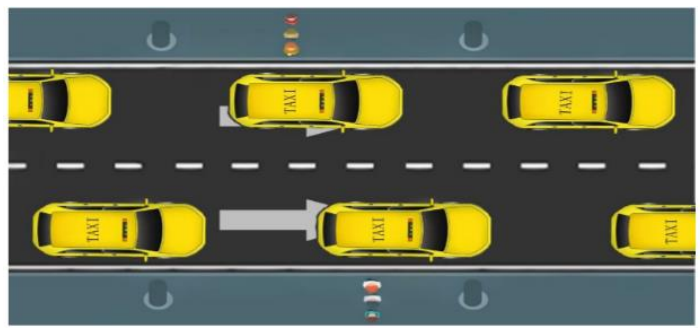

Fig. 1 Parallel lane traffic map 
It can be clearly seen from Fig. 1 that boarding points should be set on both sides of the road to ensure a balanced traffic flow in the parallel lanes. In order to improve the boarding efficiency of the boarding area, that is, to reduce the queue time of each passenger, increasing the boarding point can reduce the length of each team, but increasing the boarding point will cause the airport to invest more construction costs and management costs, so the length of passenger queues and the number of boarding points are two contradictory goals. Therefore, in this paper, the dual-objective planning model for airport study on the problem of setting up the boarding points.

\section{The establishment of Driver's choice deci- sion model}

In the two schemes, the driver receives different benefits and the length of the passenger cycle is different. We cannot compare one of these variables to reflect both the driver's gain, the driver's time cost, and the potential benefits that may be lost. Therefore, we define the ratio of the driver's income in the passenger cycle to the length of the passenger cycle as the driver's unit time, that is, time efficiency $\eta$. By comparing the time benefits $\eta_{A}$ and $\eta_{B}$ of the two

$$
Q_{B}=\overline{\omega_{s}}-2 \overline{\omega_{y}}=\overline{\omega_{s}}-2 \bar{s} m
$$

(Among them, $Q_{B}$ represents the profit obtained by a single passenger cycle driver under plan $\mathrm{B} ; \bar{\omega}_{s}$ represents the average transportation cost from the urban area to the airport; $\overline{\omega_{y}}$ represents the fuel consumption of a taxi trip; $\bar{s}$ represents the average distance from the airport to the urban area; $m$ represents the taxi journey process Fuel consumption per unit distance.)

$$
\overline{\omega_{s}}=\frac{\int_{S_{n}}^{S_{x}} \omega_{s}}{S_{x}-S_{n}}
$$

(Among them, $S_{x}$ and $S_{n}$ respectively indicate the maximum distance and the minimum schemes, and then judging the pros and cons of the schemes in different situations, the specific steps to solve the time benefits are as follows:

4.1 Analysis of plan B (Plan B is to return to the city without passengers.)

Step1: The determination of the driver's benefit in a carrying Passengers cycle

When the driver arrives at the airport, he chooses to give up waiting and return directly to the city without a load. This process does not generate any revenue for the driver. Assume that when the driver adopts this scheme, whenever he returns to the city, he can immediately receive new passengers and return to the airport, that is, regardless of the time when the driver is looking for customers in the city. At the same time, in order to reflect the no-load expenses paid by the driver for returning to the urban area without a load, the fuel consumption of gasoline is used to reflect this indicator. Therefore, the driver's income under this scheme is the fee earned by the driver from the airport to the airport minus the fuel consumption of the two round-trip taxis. The specific expression is as follows:

\section{Setp2: Determination of average prices}

Because the driver receives the uncertainty of the distance from the passenger to the airport, which makes it impossible to determine the driver's benefits, this article uses the average price from the urban area to the airport as the driver's one-time passenger fee: 
Step3: Determination of carrying passenger cycle

When the driver chooses to give up waiting to return to the city directly, the driver does not spend time at the airport at this time, just consider the time the driver spends on the way back to the city, whose expression is: $t_{B}=2 \overline{t_{s}}$
(Among them, $\bar{t}_{s}$ is the average time it takes a taxi to travel from the airport to the city)

Step4: Determination of benefits of plan B

According to the definition above, the time benefit is the ratio of the revenue of a single passenger cycle to the length of the cycle, whose expression is:

$$
\begin{gathered}
\eta_{B}=\frac{Q_{B}}{t_{B}}=\frac{\overline{\omega_{s}}-2 \overline{s m}}{2 \overline{t_{s}}} \\
\text { (Among them, } \overline{\omega_{s}}=\frac{\int_{S_{n}}^{S_{x}} \omega_{s}}{S_{x}-S_{n}} \text { ) }
\end{gathered}
$$

4.2 Analysis of plan A (Plan A is to go to the car storage pool to wait in line for passengers to return to the city.)

Step1: The determination of the driver's benefit in a carrying Passengers cycle

When the driver chooses to go to the car storage pool to wait in line for passengers, at this point the driver can earn two transport costs in a cycle, and the corresponding plan $\mathrm{B}$ also needs to pay the fuel consumption of the taxi. Therefore, the income of this plan is the two transport costs minus the fuel consumption of two taxis, the specific expression is as follows:
$Q_{A}=2 \overline{\omega_{s}}-2 \overline{\omega_{y}}=2 \overline{\omega_{s}}-2 \bar{s} m$

(Among them, $Q_{A}$ represents the profit obtained by a single passenger cycle driver under plan $A ; \overline{\omega_{s}}$ represents the average transportation cost from the urban area to the airport; $\overline{\omega_{y}}$ represents the fuel consumption of a taxi trip; $\bar{s}$ represents the average distance from the airport to the urban area; $m$ represents the taxi journey process Fuel consumption per unit distance.)

Setp2: Determination of average prices

Similar to plan B, the average price is used as the transportation cost for the driver to transport once, whose expression is:

$$
\overline{\omega_{s}}=\frac{\int_{S_{n}}^{S_{x}} \omega_{s}}{S_{x}-S_{n}}
$$

Step3: Determination of carrying passenger cycle

When the driver chooses to wait in the car storage pool, the length of the passenger cycle includes the time spent on the road and the waiting time in the storage pool, whose expression

is: $t_{A}=2 \overline{t_{s}}+t_{D}$

(Among them, $t_{D}$ is the waiting time of the driver in the car storage pool.)

Step4: Determination of waiting time

It can be known from the actual situation that the waiting time is determined by the number of queuing taxis and the number of passengers, and the queue length of the queuing taxis, that is, the existing vehicles in the car storage pool are the information that the driver can observe, so we give the waiting Expression of time: $t_{D}=\frac{l_{g}}{\mu}$

(Among them, $l_{g}$ is the number of vehicles in the car storage pool; $\mu$ is the number of vehicles that can successfully carry passengers per 
unit time, determined by the number of flights The time benefit of plan $A$ is:

arriving in a certain period.)

\subsection{Choice of plan}

In this paper, when choosing a scheme, based on the time benefits of the two schemes, the

$$
\eta_{A}=\frac{Q_{A}}{t_{A}}=\frac{2 \overline{\omega_{s}}-2 \overline{s m}}{2 \bar{t}_{s}+t_{D}} \text { (Among them, } \overline{\omega_{s}}=\frac{\int_{S_{n}}^{S_{x}} \omega_{s}}{S_{x}-S_{n}} \text { ) }
$$

decision is made by comparing the time benefits of the two schemes. Make $\eta_{A}>\eta_{B}$ :

$$
\frac{2 \overline{\omega_{s}}-2 \bar{s} m}{2 \overline{t_{s}}+t_{D}}>\frac{\overline{\omega_{s}}-2 \bar{s} m}{2 \overline{t_{s}}}
$$

Use this to judge the situation when the time benefit of plan $A$ is large. From the mathematical derivation, the relationship between the waiting

$$
t_{D}=\frac{l_{g}}{\mu}<\frac{2 \overline{\omega_{s}} t_{s}}{\overline{\omega_{s}}-2 \overline{s m}}
$$

According to the actual situation, the information This article hopes to be able to make a decision that the driver can observe is the number of vehicles in the "storage pool" and the number of flights in a certain period, but the information redirectly by directly judging the number of vehicles in the car storage pool, so slightly modify the formula (2), whose expression is:

time and each parameter can be obtained from (1). details as follows: flected by the number of flights is not intuitive.

$$
l_{g}<\frac{2 \overline{\omega_{s} t_{s}}}{\overline{\omega_{s}}-2 \overline{s m}} \mu
$$

Define $\lambda$ as the vehicle threshold for a certain period, and its expression is:

$$
\left\{\begin{array}{l}
\lambda=\frac{2 \overline{\omega_{s} \bar{t}_{s}}}{\overline{\omega_{s}}-2 \bar{s} m} \mu \\
\overline{\omega_{s}}=\frac{\int_{S_{n}}^{S_{x}} \omega_{s}}{S_{x}-S_{n}}
\end{array}\right.
$$

That is to say, this article translates the problem into just judging whether the number of vehicles in the current car storage pool is greater than the vehicle threshold at that moment. If it is greater, then choose plan B; if it is smaller,

$$
\left\{\begin{array}{l}
\eta_{B}=\frac{Q_{B}}{t_{B}}=\frac{\overline{\omega_{s}}-2 \bar{s} m}{2 \overline{t_{s}}} \\
\eta_{A}=\frac{Q_{A}}{t_{A}}=\frac{2 \overline{\omega_{s}}-2 \bar{s} m}{2 \overline{t_{s}}+t_{D}} \\
\overline{\omega_{s}}=\frac{\int_{S_{n}}^{S_{x}} \omega_{s}}{S_{x}-S_{n}}
\end{array}\right.
$$


The judgment model we have established is:

$$
\left\{\begin{array}{l}
\max f=\left\{\eta_{A}, \eta_{B}\right\} \\
\lambda=\frac{2 \overline{\omega_{s} t_{s}}}{\overline{\omega_{s}}-2 \bar{s} m} \mu \\
l_{g}<\lambda, \text { choose plan } A \\
l_{g}>\lambda, \text { choose plan } B \\
l_{g}=\lambda, \text { both }
\end{array}\right.
$$

5. The establishment of Multi-objective programming model

For the reasonable setting of the boarding point position, you need to first determine the position relationship between it and the dual-parallel lanes. After determining its position, then calculate the optimal position by using the taxi driving rules and the number of passengers a reasonable number of boarding points.

\subsection{Determine the location of the boarding point}

The location of the boarding points needs to be considered from the perspectives of safety and smooth roads. It is assumed that the boarding points are set on both sides of the parallel lane. If there is a source of passengers in one of the boarding points and the taxi is parked next to the boarding point, it will cause the subsequent taxis to block and wait. A local traffic bottleneck is formed at the boarding point, which results in a reduction in the capacity of parallel lanes and a waste of resources at the subsequent boarding point. The specific traffic diagram is shown in Fig. 2:

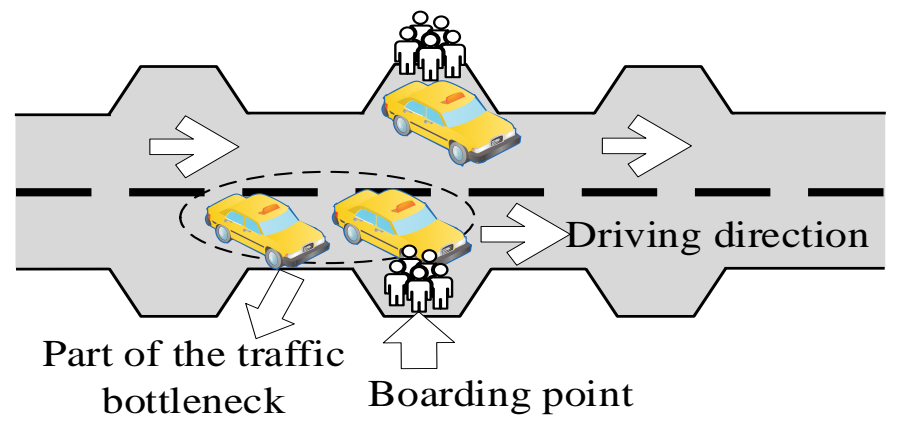

Fig. 2 Traffic diagram
Therefore, this article constructs a taxi traffic mode based on two parallel lanes, and sets the boarding point on one side of the parallel lanes. The one-way lane on this side is used as a taxi stop for picking up passengers, which is called the pick-up lane; a one-way street on the other side serves as the main road for taxis. The taxi is driven on the pick-up lane without load first. If a taxi ahead stops in the lane due to pick-up, it will immediately turn into the main road. The specific traffic diagram is shown in Fig. 3 :

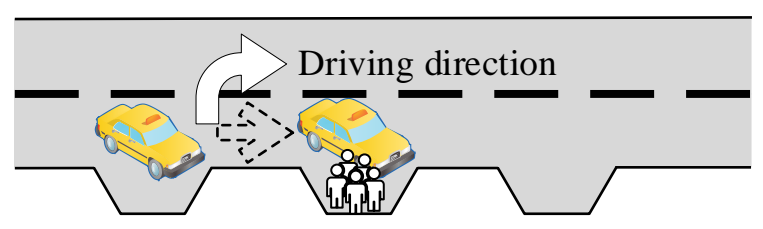

Fig. 3 Traffic diagram 
When the taxi is running on the main road with no load, if there is no taxi stop to pick up passengers in front of the boarding point, immedi- ately transfer to the pick-up lane to the boarding point for pick-up. The specific traffic diagram is shown in Fig. 4:

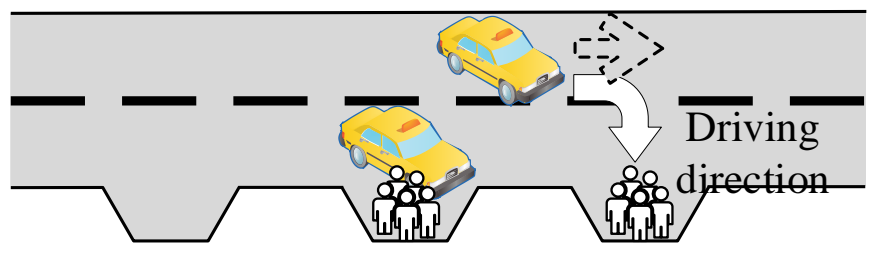

Fig. 4 Traffic diagram

Based on the above analysis, in the traffic method constructed in this article, except for taxis that need to stop and wait when picking up passengers, the taxis are driven at normal speed in other cases, and will not stop or slow down due to other factors, ensuring smooth roads. And it will not cause local traffic bottlenecks to waste the resources of the boarding points.

The taxi passing mode based on the two parallel lanes constructed above has determined the relative positions of the parallel lanes and the boarding points. Based on this, the number of boarding points also needs to be determined.

\subsection{Construction of queuing theory model}

In the taxi passing mode mentioned above, except for the taxi need to stop and wait for passengers, the taxi will travel at a normal speed. If the taxi traffic is regarded as a service organization, the traffic and passengers entering the boarding area together constitute a service system, and each boarding point is a service desk ${ }^{[4-}$ 5], then the service system meets the $M / M / C / \infty / \infty$ model in queuing theory, that is, this problem can be solved based on this model and theory ${ }^{[6-8]}$.

\subsection{Determination of objective function}

$$
\begin{aligned}
\tau_{n} & =\tau,(n=0,1,2, \cdots) \\
\theta_{n} & =\left\{\begin{array}{l}
n \theta, \theta=1,2, \cdots, c \\
c \theta, n=c, c+1, \cdots
\end{array}\right.
\end{aligned}
$$


(Among them, $\tau$ represents the number of passengers who arrive at the boarding point within unit time; $\theta$ represents the number of passengers who can successfully board the unit within unit time.)

Let $\rho_{c}=\frac{\rho}{c}=\frac{\tau}{c \theta}$, when $\rho_{c}<1$, it can be proved mathematically:

$$
p_{0}=\left[\sum_{n=0}^{c-1} \frac{\rho^{n}}{n !}+\frac{\rho^{c}}{c !\left(1-\rho_{c}\right)}\right]^{-1}
$$

The average team length is $l_{q}$ :

$$
l_{q}=\frac{p_{0} \rho^{c} \rho_{c}}{c !\left(1-\rho_{c}\right)^{2}}
$$

(Among them, $p_{0}$ represents the probability when no passengers are waiting to get on the bus; $\rho_{c}$ represents the system service intensity when the number of service desks is $c ; c$ represents the number of boarding points.)

So we take the minimum quotient of the average team length and the number of passengers who can successfully board a car as the objective function, and its expression is: $\min f=\frac{l_{q}}{\theta}$

Step2: When setting the number of boarding points, it is not only necessary to maximize the overall boarding efficiency, but also necessary to consider the cost of the construction of boarding points from the perspective of the airport. In other words, it is better to make the number of boarding points as small as possible on the premise of ensuring the overall boarding efficiency, and its expression is: $\min g=c$

\subsection{Determination of constraint conditions}

Step1: A series of formulas such as the average team length in this article are based on the condition of service intensity $\rho_{c}<1$, because when $\rho_{c}>1$, it will result in an infinitely long team, therefore, we need: $\rho_{c}=\frac{\tau}{c \theta}<1$

Step2: The number of boarding points will not be infinite. It will be affected by factors including the length of the lane, the length of the body, and the safe distance of the vehicle, there will be a critical threshold, its expression is $c<\gamma$.

Step3: The number of boarding points must be a positive integer, its expression is: $c \in N^{+}$.

In summary, Multi-objective programming model we built is:

$$
\begin{gathered}
\min f=\frac{l_{q}}{\theta} \\
\text { s.t. }\left\{\begin{array}{l}
\min g=c \\
l_{q}=\frac{p_{0} \rho^{c} \rho_{c}}{c !\left(1-\rho_{c}\right)^{2}} \\
p_{0}=\left[\sum_{n=0}^{c-1} \frac{\rho^{n}}{n !}+\frac{\rho^{c}}{c !\left(1-\rho_{c}\right)}\right]^{-1} \\
\rho=\frac{\tau}{\theta} \\
\rho_{c}=\frac{\rho}{c}<1 \\
c<\gamma \\
c \in N^{+}
\end{array}\right.
\end{gathered}
$$

\section{Case study of models}

In order to ensure the practicability and reliabili- 
ty of the model, so that Driver's choice decision model and Multi-objective programming model given in this article have practical significance, and can provide reasonable suggestions for taxi drivers and airports, so that the model has good application, this article will choose Shanghai Hongqiao Airport Research object. By querying the data, we collected the airport throughput of Shanghai Hongqiao Airport in each month of 2018, the number of flights at each time of day during the peak season, and the taxi pricing method and corresponding parameters in Sh- anghai. Through the data analysis of the collected data, to determine the parameter values of the model at Shanghai Hongqiao Airport.

As the monthly passenger throughput of a single airport is difficult to find, the monthly percentage of air throughput of Shanghai Hongqiao Airport is considered equal to the monthly percentage of air throughput of the eastern region. The data can be used to calculate the monthly passenger throughput of Shanghai Hongqiao Airport. The calculated data is plotted into a histogram as shown in Fig. 5:

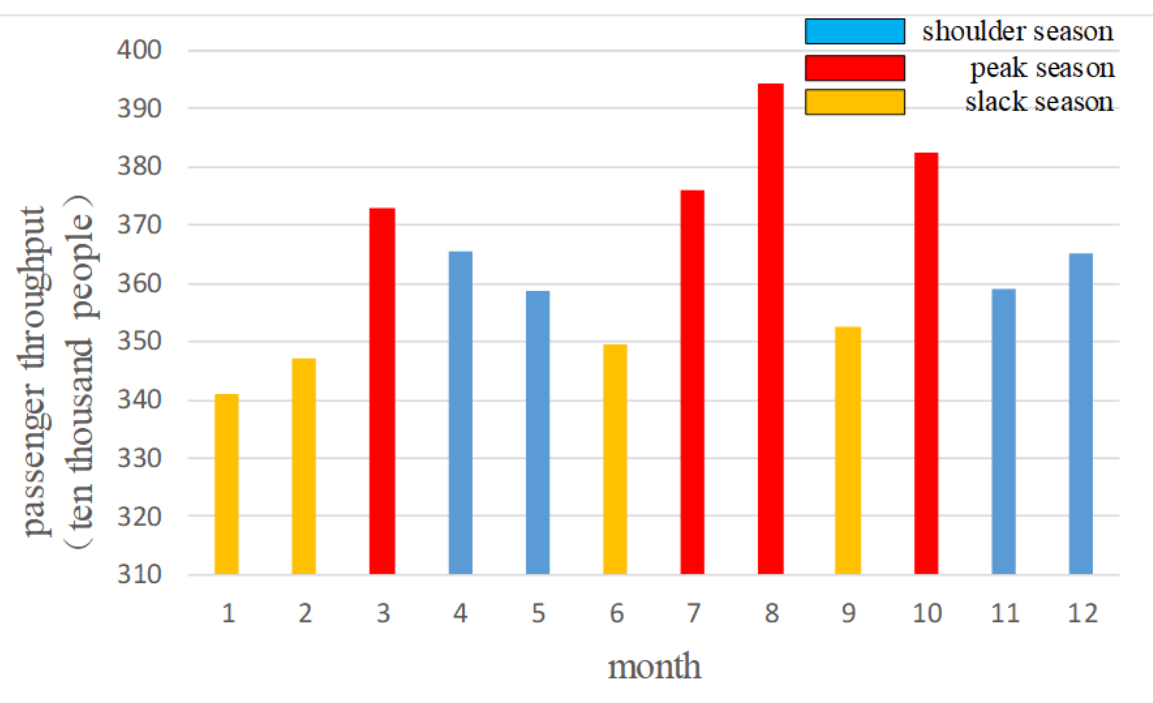

Fig. 5 Histogram of monthly passenger throughput

In addition, the number of flights is analyzed, and a pie chart of the number of flights in

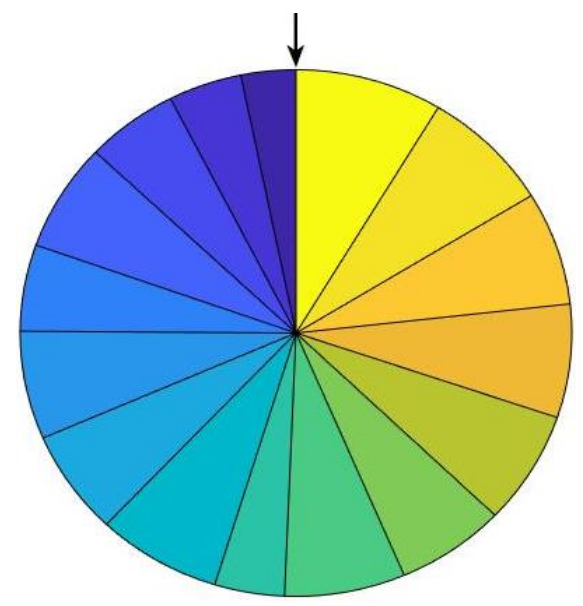

Fig. 6 (From where the arrow points counterclockwise, different times of the day can be drawn as shown in Fig. 6:

\begin{tabular}{cc|cc}
\hline \hline $\begin{array}{c}\text { Serial } \\
\text { number }\end{array}$ & $\begin{array}{c}\text { Number of } \\
\text { flights }\end{array}$ & $\begin{array}{c}\text { Serial } \\
\text { num- } \\
\text { ber }\end{array}$ & $\begin{array}{c}\text { Number } \\
\text { of flights }\end{array}$ \\
(1) & 29 & $(9)$ & 37 \\
(2) & 39 & $(10$ & 64 \\
(3) & 49 & 11 & 58 \\
(4) & 60 & 12 & 63 \\
(5) & 48 & 13 & 63 \\
(6) & 60 & $(14)$ & 63 \\
(7) & 58 & 15 & 69 \\
(8) & 66 & 116 & 78 \\
\hline
\end{tabular}

each time 
According to the above figure, the number of flights in each time period can be simply read. It can be found that there are only a few times when the number of flights is less than 50 and most times the number of flights is 60 or more, and showed a slowly rising trend.

\subsection{The result analysis of Driver's choice de- cision model}

According to Driver's choice decision model given in this article, combined with the abovementioned data collected about Shanghai Hongqiao Airport, MATLAB software was used to calculate the vehicle threshold in the car storage pool at each moment of the daily average of each month ${ }^{[9]}$. Draw into a three-dimensional perspective, as shown in Fig. 7.
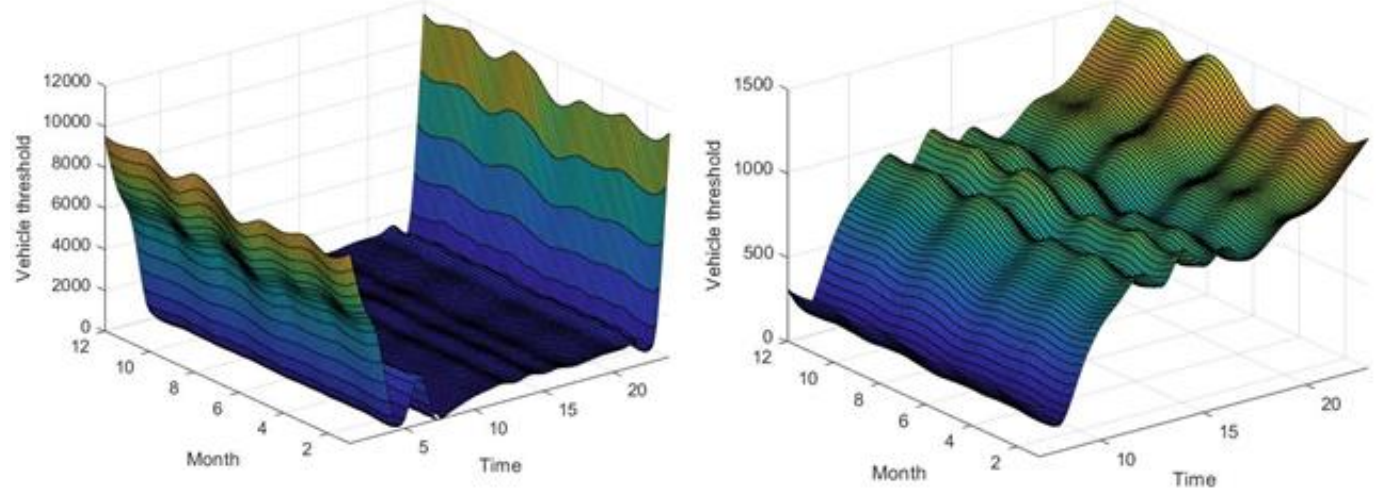

Fig. 7 three-dimensional perspective for the vehicle threshold

In the two pictures above, the left picture is the vehicle threshold value in the car storage pool from 00: 00-24: 00, it can be clearly seen that the value of the number of queued cars in the car storage pool at 00: 00-6: 00 every day is distorted, that is, deviating from the specific situation of real life, the data for this time period is invalid, analyzing the reason, this kind of phenomenon occurs because the maximum number of critical queues in the car storage pool is not limited in the model. Therefore, in order to truly and accurately reflect the characteristics of the vehicle threshold value in the car storage pool at each time of the day, the number of queuing vehicles in the car storage pool at 7:0023: 00 daily is selected to be drawn as shown graphics. Observing this chart, we can clearly see that 7: 00-9: 00 daily is the quiet period of the airport, and fewer passengers arrive at the airport; 9: 00-18: 00 daily is the steady period of the airport and arrive at the airport, and the number of passengers tends to be dynamically balanced; 18: 00-23: 00 is the active period of the airport every day, and more passengers arrive at the airport.

\subsection{Sensitivity analysis}

As mentioned above, the threshold for the number of taxis is not necessarily constant. The number of flights, the average distance from the airport to the city center, and the fuel price per kilometer are three very important parameters that have a direct impact on the vehicle threshold for each period. In order to understand whether the changes in these three parameters lead to drastic changes in results, a sensitivity analysis is performed here for the number of flights per time period, the average distance from the city center, and the fuel price per kilometer.

In order to simplify the problem, we assume here that the variables in each time period are increased or decreased in the same proportion, that is, set the variable to be $k$ times the original value, redefine the constraint, repeat the above 
solution process, and find different $k$ to the vehicle threshold value.

May wish to take $\pm 1 \%$ as the amount of change,
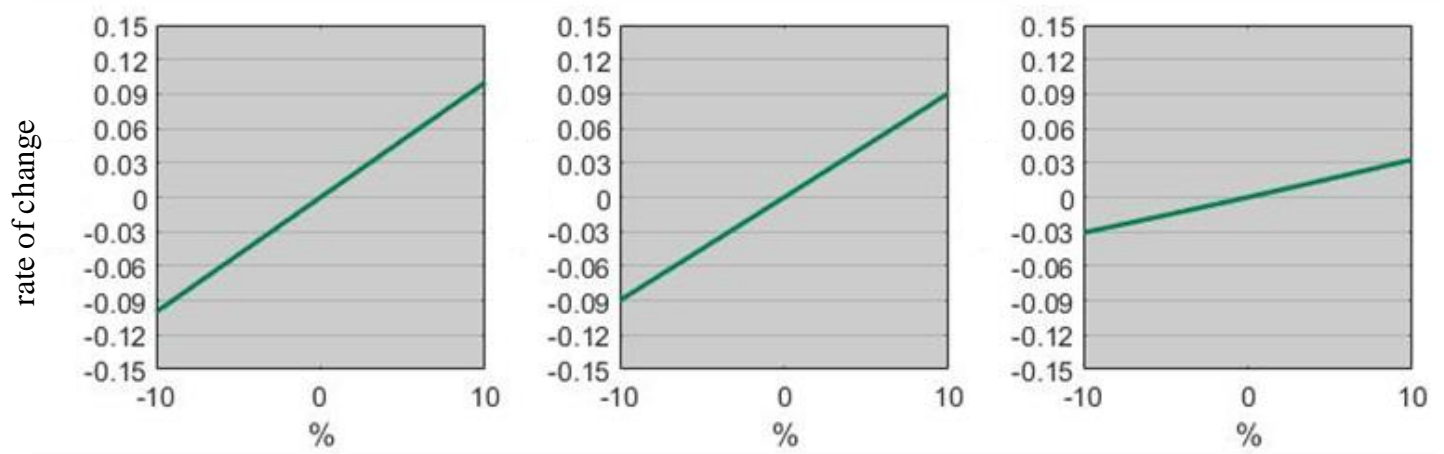

Fig. 8 Line chart of number of flights, average distance from the city center, fuel price sensitivity per kilometer

By analyzing the above figure, we can know that the number of flights, the average distance from the city center and the result change are relatively high, and the sensitivity is high, that is, the model is highly dependent on these two factors. The fuel price per kilometer is poorly correlated with the change in results, and the sensitivity is low, that is, the model is less dependent on this factor.

Through the case study of Shanghai Hongqiao Airport to test the model, we found that the results are consistent with the actual situation, that is, the model has passed the rationality test, and the model's dependence on the parameters

$$
\left.d=\left[\sum_{i=1}^{2}\left(h_{i}(x)-h_{i}^{*}\right)^{p}\right]^{\frac{1}{p}} \quad \text { (Among them, } p \text { is bound norm, usually take } p=2 .\right)
$$

For this model, substituting relevant data, and using MATLAB software to obtain the ideal point of the model $H^{*}=\left(f^{*}, g^{*}\right)=(0.0652,3)$, and the minimum number of boarding points is 3 , at this time the minimum time required for each passenger to wait is 2.044 minutes.

\section{Advantages of the model}

(1) In the Driver's choice decision model, a method of mechanism analysis is used to find not only the relevant factors that affect the taxi driver's decision-making, but also the mechanism and relationship between the influencing factors. Unlike data analysis, only the weights of relevant factors can be is within the tolerance range, that is, the model can Reasonable advice for taxi drivers at most airports.

\subsection{The result analysis of Multi-objective programming model}

Because the above optimization model is multiobjective optimization, the two objective functions cannot reach the optimal at the same time, so it is difficult to solve the model with general methods. Therefore, this paper uses the evaluation function method to analyze the model ${ }^{[10]}$. Select the ideal point method, let $H^{*}=\left(h_{1}{ }^{*}, h_{2}{ }^{*}\right)$ is the ideal point of the model, let:

found.

(2) Driver's choice decision model skillfully converts the judgment of the time benefit of the two schemes into a comparison of the number of vehicles in the pool and the threshold of the vehicle volume, which greatly simplifies the selection strategy.

(3) When establishing Driver's choice decision model, the taxi driver's decision-making is made to every moment, so that the decision model has time dynamics, so that the driver's decision is more accurate.

(4) When considering the influencing factors of taxi driver's decision-making, considering the competition of subway, bus and other 
Hairui Zhang et al., IJSSM, 2020; 3:7

means of transportation, the probability of the taxi being selected is obtained based on the checked data, which makes the decision result closer to the reality.

(5) Model two uses multi-objective programming and simultaneously optimizes the two objective functions as much as possible, making the results more reasonable than the results of single-objective programming.

\section{Shortcomings of the model}

(1) In order to simplify the model, when considering the distance between Shanghai Hongqiao Airport and the city, only the distance between Hongqiao Airport and East China University of Science and Technology in Xuhui District, Shanghai was selected to express the average distance.

(2) Multi-objective programming model is only applicable when the number of taxis in the car storage pool is very large, which has certain limitations.

\section{Promotion of the model}

First of all, in modern scientific decision-making, the methods of natural science and mathematical tools are often used to establish the relationship formulas and models between decision variables to reflect the essence of decisionmaking problems and simplify complex decision-making problems. In addition, the multi-objective programming model is not affected by the data distribution, sample size, and number of indicators. It is flexible, convenient, and highly reliable, and can be used in multiple industries such as industrial production and economic returns. The queue theory following the Poisson process can also be widely used in transportation systems, port berth design, machine maintenance, inventory control and other service systems.

\section{Conclusion}

In this paper, Driver's choice decision model and Multi-objective programming model are established to address the choice decision problems faced by airport taxi drivers after sending passengers to the airport and the setting-up point of the airport boarding point. The data of Shanghai Hongqiao Airport and Shanghai Taxi were collected, and the specific options for taxi drivers at this airport were given. It was found that the model was highly dependent on the two factors of flight frequency and the average distance from the airport to the city center. The dependence on the fuel cost per kilometer is relatively low, and for the peak season of passengers at Shanghai Hongqiao Airport, we found that evenly setting up three boarding points beside the dual parallel lanes in the airport's passenger area can make the overall boarding efficiency is the highest. This article provides airport taxi drivers with a more reasonable decision-making method. The method is simple and easy to judge, protects the benefits of airport taxi drivers, and solves the inefficiency of airport operations caused by airport passengers waiting for taxis for too long This problem has far-reaching significance for the systematization of airport taxi queuing.

\section{References}

1. Zhihong $\mathrm{Hu}$, Wei Dong, Liu Cao, Zhong Gao, Zhiyong Lu, Jun Lv, Hongbiao Huang, Feifan Gu. Construction and implementation of intelligent matching management system for taxis in large transportation hubs.

2. Hailin Zhang. Research on Terminal Lane System Based on Passenger Service and Airport Management_— Taking Xianyang Airport as an Example.

3. Tiehan Xie. Problems and Countermeasures in Vehicle Dispatching Management [J] .The Think Tank Era.

4. Jian Sun. Modeling and Simulation of Land-side Passenger Service Resources for Aviation Hub Based on Queuing Theory.

5. Shoukui Si, Zhaoliang Sun. Mathematical Modeling Algorithms and Applications, Beijing: $\mathrm{Na}-$ tional Defense Industry Press, 2015.2.

6. Zhuohao You. Research on Cloud Resource Scheduling Strategy Based on M / M / n Queueing Model [D] .University of Electronic Science and Technology of China, 2014.

7. Frank R. Giordano, William P. Fox, Steven B. Horton. Mathematical Modeling, Beijing: Mechanical Industry Press, 2014.6.

8. Qiyuan Jiang, Jinxing Ye. Mathematical Model (Fifth Edition), Beijing: Higher Education Press, 
1986.6.

Hairui Zhang et al., IJSSM, 2020; 3:7

9. Zhen Liu, Wenbiao Liu. Island-ship Integrated Cooperative Air Defense Dynamic Decision Model and Algorithm [J] .Command Control and Simulation, 2019, 41(01):20-25.

10. Yuhua Zeng, Zheng Peng. An Inexact Alternating Direction Method for Solving Bi-Objective Programming $[\mathrm{J}]$.Journal of Operations Research, 2010, 14(04):121-128. 